LOS DISCURSOS EN TORNO A LA SEXUALIDAD. SU INTERNALIZACIÓN Y REPRODUCCIÓN A TRAVÉS DE LAS PRÁCTICAS EDUCATIVAS. PÁginas 201-214 en Revista de la Escuela de Ciencias de la Educación, año 11, nÚmero 10, enero a diciembre de 2015. ISSN 1851-6297. ISSN EN LINEA 2362-3349.

\title{
LOS DISCURSOS EN TORNO A LA SEXUALIDAD. SU INTERNALIZACIÓN Y REPRODUCCIÓN A TRAVÉS DE LAS PRÁCTICAS EDUCATIVAS
}

\author{
Por Jorgelina Andrea Marozzi* \\ Ministerio De Educación De Córdoba, Argentina. \\ jomarozzi@yahoo.com.ar
}

Recibido: 27/06/2014 Aceptado: 11/08/2014

\section{Resumen}

En los últimos siglos, los discursos médicos y morales se han erigido como discursos validos o "verdaderos" en torno a la sexualidad. Con la modernidad comienzan a desplegarse dispositivos específicos de saber y de poder, a fin de disciplinar los cuerpos y modelar la sexualidad. Estos dispositivos se encarnan en normas, tradiciones, leyes jurídicas, prácticas escolares, y también se encarnan en los sujetos construyendo subjetividades. El docente, atravesado por estos discursos va configurando su propio posicionamiento, internalizando un determinado modo de pensar, decir, sentir y actuar en torno a la sexualidad, reproduciendo en sus prácticas el discurso hegemónico.

En los últimos años, asistimos a una época de cambios sociales y culturales vertiginosos, con el surgimiento de un Estado que promueve políticas de reconocimiento y garantía de derechos a actores sociales históricamente postergados, y la sanción de una ley de educación sexual integral. Estas nuevas condiciones sociales entrarían en tensión con las concepciones docentes, interpelando a los mismos a repensar las formas tradicionales en que se ha encarado la educación sexual en las escuelas.

El presente trabajo no constituye una verificación empírica de la tesis expuesta sino que muestra las elaboraciones teóricas que llevaron a su construcción.

\footnotetext{
Licenciada y Profesora de Psicología (Universidad Nacional de Córdoba). Actualmente finalizando la Tesis de la Maestría en Ciencias Sociales (Facultad de Derecho y Ciencias Sociales de la Universidad Nacional de Córdoba). Integrante del Equipo Técnico del Programa Provincial de Educación Sexual Integral del Ministerio de Educación de la Provincia de Córdoba. Docente del Seminario Educación Sexual Integral del Instituto Provincial de Educación Física (IPEF) de la Universidad Provincial de Córdoba.
} 
Revista de la Escuela de Ciencias de la Educación, año 11, número 10, enero a diciembre de 2015. Páginas 201-214. ISSN 1851-6297. ISSN EN LINEA 2362-3349. LOS DISCURSOS EN TORNO A LA SEXUALIDAD. SU INTERNALIZACIÓN Y reproducCión a través de LAS pRÁcticas educativas. Jorgelina Andrea MarozZI

\title{
Palabras clave:
}

Dispositivo de sexualidad - Discurso hegemónico - Proceso de subjetivación Posicionamiento docente - Educación sexual.

\begin{abstract}
In the last few centuries, medical discourses and morales have emerged as valid or "real" discourses on sexuality. With modernity are beginning to deploy specific devices of knowledge and power, to disciplinary bodies and shape sexuality. These devices are embodied in rules, traditions, laws, legal, school practices, and is also embodied in the subject building subjectivities. The teacher, crossed by these discourses is configuring their own positioning, internalizing a certain way of thinking, say, feel, and act on sexuality, playing in their practices the hegemonic discourse. In recent years, we are witnessing an era of rapid social and cultural changes, with the emergence of a State that promotes policies of recognition and guarantee of rights to historically neglected social actors, and the enactment of a comprehensive sex education law. These new social conditions would be in tension with the conceptions of teaching, challenging them to rethink the traditional ways in which it has faced sexual education in schools.

This work is not an empirical verification of the exposed thesis but showing the theoretical elaborations that led to its construction.
\end{abstract}

\section{Key words:}

Device of sexuality - Hegemonic discourse - Process of subjectivation - Teaching position - Sex education.

\section{Introducción}

La importancia de recibir educación sexual en forma sistemática en las escuelas de nuestro país fue en aumento en las últimas décadas; y a partir del 2006, con la sanción de la Ley 26.150, acceder a Educación Sexual Integral (ESI) a lo largo de todos los niveles del sistema educativo es un derecho. Desde su sanción hasta hoy, y aún antes de la misma, distintos actores sociales han manifestado posiciones heterogéneas al respecto, dejando entrever, además, una lucha de poderes y disputas sobre qué significa y quiénes tienen el saber sobre la Educación Sexual. Como sostiene Foucault, el conocimiento -de las cosas, de una determinada realidad- es "inventado" y resultado de una confrontación. Tal como lo expresa, "El conocimiento sólo puede ser una violación de las cosas a conocer y no percepción, reconocimiento, identificación de o con ellas" (1996, pp. 24). Entonces, partimos de considerar que la visión social que se tiene sobre la sexualidad es producto de una construcción histórica, una construcción que se genera en un sistema de relaciones que pujan entre sí por el control de la definición de la realidad. Por esto es importante ver qué discurso se ha ido construyendo (e imponiendo) sobre la sexualidad. Y para recuperar la historicidad, es lícito preguntarse ¿Quiénes hablan de sexualidad? 
Revista de la Escuela de Ciencias de la Educación, año 11, número 10, enero a diciembre de 2015. Páginas 201-214. ISSN 1851-6297. ISSN EN LINEA 2362-3349. LOS DISCURSOS EN TORNO A LA SEXUALIDAD. SU INTERNALIZACIÓN Y

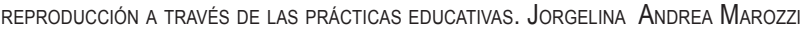

¿Qué dicen? ¿Qué callan? ¿Qué instituciones difunden lo que se dice? Esto permitirá entender qué discursos imperan en las prácticas pedagógicas con relación a lo sexual, cuáles se imponen, qué enfoques o qué perspectivas se entronizan como "verdaderas".

Subyace a este planteo, la convicción de que en las prácticas de enseñanza en torno a la educación sexual, los docentes reproducen sus propias concepciones o representaciones de la sexualidad que han internalizado a través del proceso de socialización, y éstas, estarán imbuidas de temores, mitos y prejuicios sexistas que reproducen relaciones inequitativas de género. Además, la enseñanza hará hincapié en la función biológica de la reproducción, ligando el ejercicio de la sexualidad a aspectos relacionados a la salud e imbuido de preceptos morales.

El presente trabajo no intenta constituirse en una verificación empírica de la tesis expuesta, tan solo intenta mostrar las elaboraciones teóricas que llevaron a la construcción de la misma. Así se recurrirá a los escritos de Foucault a fin de mostrar los discursos acerca de la sexualidad que han logrado imponerse a través del tiempo, y a los aportes del interaccionismo simbólico y de la teoría de Pierre Bourdieu a fin de pensar en cómo estos discursos se incorporan en los sujetos docentes produciendo subjetividades y reproduciéndose a través de las prácticas educativas. Estas producciones teóricas se constituyen en los referentes conceptuales de un trabajo de investigación en curso, que indaga acerca de la posibilidad de modificación de las concepciones docentes a partir de la participación en procesos de capacitación en Educación Sexual Integral.

\section{Los discursos sobre la sexualidad}

Las investigaciones sobre la sexualidad cobran especial relevancia en la década de 1970 con la publicación de Historia de la Sexualidad de Michael Foucault. Si seguimos al autor a través de sus reflexiones podemos admitir que los griegos en la antigüedad aceptaban mucho más fácilmente que los cristianos de la edad media o los europeos de la modernidad ciertos comportamientos sexuales, y estaba menos preestablecido lo permitido y lo prohibido, lo normal o lo anormal. En la antigüedad, no se trataba de reprimir el deseo sexual, no existía una legislación universal que determinara los actos prohibidos y permitidos sino sugerencias a fin de estilizar la conducta, prescribiendo una manera de "usar los placeres" que propiciara la austeridad sexual y el dominio de sí. Por el contrario, en la moral sexual del cristianismo, la problematización en torno a ciertas temáticas comienza a transformarse en prescripción, visualizándose al placer en sí mismo como negativo. El acto sexual comienza a ser considerado algo pecaminoso, oscuro, y no se le concede legitimidad sino dentro del matrimonio. 
Revista de la Escuela de Ciencias de la Educación, año 11, número 10, enero a diciembre de 2015. Páginas 201-214. ISSN 1851-6297. ISSN EN LINEA 2362-3349. LOS DISCURSOS EN TORNO A LA SEXUALIDAD. SU INTERNALIZACIÓN Y REPRODUCCIÓN A TRAVÉS DE LAS PRÁCtICAS EDUCATIVAS. JORgELINA ANDREA MAROZZI

Siguiendo a Foucault en La voluntad del saber nos muestra de qué manera, en los últimos siglos, la sexualidad fue "cuidadosamente encerrada" si tenemos en cuenta la libertad con la que contaba en la época clásica, y si bien, parecería que se han impuesto represiones a lo sexual, en realidad se han multiplicado los discursos sobre el sexo. Si bien es cierto que hay control, censura en el vocabulario, se establecen los lugares donde no se habla (entre padres e hijos, educadores y alumnos) y donde es necesario hablar (prácticas confesionales, escucha clínica); en realidad se establece un "nuevo régimen de los discursos", no se dice menos sino de otro modo, son otras personas quienes lo dicen, a partir de otros puntos de vista, para producir otros efectos. Podemos ver entonces, como el poder permite reprimir, controlar el discurso de los otros (lo que no se puede decir) y reservarse el monopolio de la producción y definición de sentido (lo que es correcto decir)

En la modernidad, la conducta sexual de la población es tomada como objeto de análisis y surge la necesidad de reglamentar el sexo mediante el control de la natalidad, la edad de matrimonio, las prácticas anticonceptivas. En el siglo XVIII, se puso en acción todo un aparato para producir sobre el sexo "discursos verdaderos", instalándose un dispositivo de sexualidad, donde multiplicidad de discursos funcionan en instituciones diferentes, regulando el cuerpo y sus placeres, a través de una serie de intervenciones y controles reguladores. La sexualidad se liga a la reproducción y excluye las prácticas que no tienen la generación como fin, se alienta la monogamia heterosexual, no se tolera la exteriorización de los deseos instintivos y se multiplican las condenas judiciales por las perversiones. Dice Foucault, "Por mediación de la medicina, la pedagogía y la economía, hizo del sexo no solo un asunto laico sino un asunto de Estado; aún más: un asunto en el cual todo el cuerpo social, y casi cada uno de sus individuos, era instado a vigilarse" (2002, pp. 141)

Así, el dispositivo de sexualidad erigido en la modernidad, buscó reglamentar institucionalmente la sexualidad, surgiendo una nueva tecnología de poder y estrategias empleadas por el biopoder para disciplinar a los sujetos. La conducta sexual de la población es tomada como objeto de análisis y se torna en "tema de operaciones políticas, de intervenciones económicas (mediante incitaciones o frenos a la procreación), campañas ideológicas, de moralización o de responsabilización" (2002, p. 177)

Durante el siglo XIX, el sexo se inscribe en dos registros de saber muy distintos, por un lado, una biología de la reproducción, con hincapié en los aspectos fisiológicos a través de un "discurso científico", y una medicina de la sexualidad ligada a aspectos morales o políticos, caracterizada, dice Foucault, con una "voluntad de no saber", de ocultar, de silenciar. Los discursos médicos y morales se han erigido como los discursos validos o "verdaderos" sobre el sexo en los últimos siglos. 
Revista de la Escuela de Ciencias de la Educación, año 11, número 10, enero a diciembre de 2015. Páginas 201-214. ISSN 1851-6297. ISSN EN LINEA 2362-3349. LOS DISCURSOS EN TORNO A LA SEXUALIDAD. SU INTERNALIZACIÓN Y

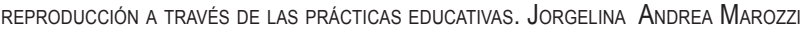

En consonancia con esto, si analizamos qué discursos se han instituido como verdaderos en relación a la sexualidad en una institución social como la escuela, podemos determinar, siguiendo los planteos de Graciela Morgade (2006) dos modelos dominantes en los programas de educación en la sexualidad en diferentes países del mundo:

1. Un modelo biologista, el cual considera que en la escuela se abordan cuestiones de sexualidad si se estudia la anatomía de la reproducción. La sexualidad queda reducida a genitalidad, separada de las emociones o de las relaciones humanas que le dan sentido al uso del cuerpo biológico.

2. Un modelo moralizante, que enfatiza las cuestiones vinculares y éticas que sustentan las expresiones de sexualidad, desde un sistema normativo (el "deber ser").

Estos enfoques parcializan la cuestión de la sexualidad, tendiendo a silenciar las realidades de niños/as, jóvenes y adultos/as y, por acción u omisión terminan reforzando las relaciones de poder hegemónicas.

Al desplegarse entonces dispositivos específicos de saber y de poder, a fin de controlar, imponer un discurso válido o verdadero respecto al sexo, el sujeto va siendo producido por los dispositivos. Se construye una forma de ser mujer, de ser varón, un modelo de familia, al que se le da un carácter de natural y a-histórico. Lo "femenino" se constituye en un lugar de subordinación y se lo asimila a lo pasivo, lo emocional, lo privado, mientras que lo "masculino" a lo activo, lo racional, lo público. Se establecen jerarquías de valor sexual donde la sexualidad "buena", "normal" y "natural" sería idealmente heterosexual, marital, monógama, reproductiva y no comercial" y toda manifestación diferente es considerada "mala", "anormal" o "antinatural" (Rubin, 1984). Estas significaciones, se plasman, no solamente en las normas y tradiciones, en leyes jurídicas, en los programas escolares, sino también en los cuerpos, construyendo subjetividades.

Para explicar de qué manera, los discursos de una época se inscriben en los sujetos conformándolos como tal, construyendo sus identidades, recurriré a los aportes de los teóricos del interaccionismo simbólico y a la teoría de Pierre Bourdieu.

\section{De lo social a lo individual. El proceso de subjetivación}

El proceso de subjetivación, aquel que transforma a un individuo en sujeto, comienza cuando este "asume" el mundo en el que ya viven otros, con sus reglas, sus valoraciones, sus significaciones. La vida cotidiana se aprende como una realidad dada, ordenada, objetivada, es decir, con objetos que son designados y significados desde antes que el individuo nazca. Los otros significantes mediatizan el mundo, seleccionan aspectos del mundo según la posición que ocupan en la estructura social y también en función de sus trayectorias individuales, el mundo social aparece entonces "filtrado" para el individuo, y 
Revista de la Escuela de Ciencias de la Educación, año 11, número 10, enero a diciembre de 2015. Páginas 201-214. ISSN 1851-6297. ISSN EN LINEA 2362-3349. LOS DISCURSOS EN TORNO A LA SEXUALIDAD. SU INTERNALIZACIÓN Y REPRODUCCIÓN A TRAVÉS DE LAS PRÁCTICAS EDUCATIVAS. JORgELINA ANDREA MaROzZI

es importante tener en cuenta, tal como lo señalan Berger y Luckmann (1989) que el niño no internaliza el mundo como uno de los posibles, sino como el mundo, el único que existe, y por ello el mundo internalizado en la socialización primaria se implanta con firmeza.

Desde que tenemos interacción se incorpora un sentido común compartido, un sentido común acerca de la realidad que hace que veamos como natural algo que en realidad es construido. Este acervo de conocimientos, estas estructuras de sentido aprendidas por las experiencias subjetivas en la vida cotidiana, desde las cuales se percibe la realidad y se actúa, no son captadas por la conciencia, y por lo tanto se presentan como conocimientos "obvios", no problemáticos.

Por otra parte, Bourdieu desarrolla el concepto de habitus para referirse a aquellos principios de percepción y apreciación de la realidad que se constituyen a lo largo de la historia del sujeto, por la interiorización del mundo objetivo a partir de una posición diferencial en el espacio social. Los agentes son distribuidos en el espacio social global según el volumen de capital que poseen bajo diferentes especies y según la estructura de su capital económico y cultural, y es a partir de la posición que ocupan en este espacio, que perciben la realidad social, por lo tanto las representaciones de los agentes varían según su posición y según su habitus que adquieren a través de la experiencia duradera de una posición en el mundo social.

Sabemos que el mundo social puede ser construido de diferentes modos según la posición, y que los miembros de una misma clase y de una misma época, están expuestos a experiencias similares, y por lo tanto construyen esquemas de percepción y de acción similares.

Ahora, podemos analizar si el grupo de docentes, que son quienes están encargados de la enseñanza de cuestiones vinculadas a la sexualidad en la escuela, se constituye como un grupo homogéneo o no. Algunos estudios (Tenti Fanfani, 2005) marcan que si bien existe gran heterogeneidad en los docentes (en edad, formación) hay algo que los caracteriza y es su posición relativa en la estructura social. A través de indicadores objetivos como el ingreso y la procedencia social, y de factores subjetivos como la propia imagen de clase que los docentes tienen, la auto percepción en términos de condición de pobreza, entre otros, llega a la conclusión de que los profesores ocupan una posición intermedia en la estructura de la sociedad, como representantes de las clases medias. Si tenemos en cuenta que los agentes, tienen tantas más propiedades en común cuanto más próximos estén en el espacio social, y tantas menos cuanto más alejadas, podemos decir que los y las docentes constituyen un grupo con maneras de pensar, de valorar, de apreciar similares. Debido a la singularidad de las trayectorias sociales, el habitus individual, el estilo personal de cada docente, reflejaría una diversidad en la homogeneidad, sería solo una desviación respecto al estilo propio de una época o una clase. 
Revista de la Escuela de Ciencias de la Educación, año 11, número 10, enero a diciembre de 2015. Páginas 201-214. ISSN 1851-6297. ISSN EN LINEA 2362-3349. LOS DISCURSOS EN TORNO A LA SEXUALIDAD. SU INTERNALIZACIÓN Y

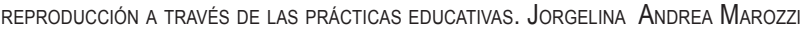

A partir de aquí podemos pensar: ¿Qué sentido común compartido por los docentes existe acerca de la sexualidad? ¿Qué es aquello que aparece como evidente y no se cuestiona? ¿Qué representaciones o concepciones sobre la sexualidad se han construido? ¿Qué prácticas de educación sexual imperan? ¿Qué posicionamiento asumen?

Los educadores, además de sus trayectorias personales, "se constituyen" como tales en el seno de las instituciones en las que se forman, con conocimientos y actitudes vinculados a las disciplinas que enseñan y a las específicas de su formación pedagógica, con representaciones sobre la educación y la realidad social. Estas instituciones funcionan en lo que Bourdieu denomina el "campo social", campo atravesado por luchas de poder en relación a los capitales en juego, por lo que la formación de los educadores va a estar marcada, sobrederminada diría Raúl Ageno (2000), social e institucionalmente, según hayan sido los "lugares" de su formación, por los capitales disponibles en los mismos, su circulación y las posibilidades de apropiación de cada sujeto.

Cabe aclarar que la incidencia de las condiciones sociohistóricas no es lineal ni mecánica, el sujetamiento a la estructura social no es total ni absoluto sino que está vinculado también con las vicisitudes de la historia personal de cada sujeto en relación con la cual se estructura una subjetividad que tiene un particular modo de leer/interpretar los discursos sociales que circulan en la sociedad. Para el psicoanálisis, el sujeto se constituye atravesado por el lenguaje, con las marcaciones con que los otros, adultos significativos, lo significan. Entonces, el lugar desde el cual lee/interpreta la realidad se constituye en función de la manera en que el sujeto ha metabolizado los discursos familiares, institucionales y sociales que lo han atravesado en su vida. De esta manera, en la constitución de su subjetividad interviene un registro que queda en otra escena, la del inconciente, desde donde incidirá en sus representaciones, en su manera de pensar y de actuar. Es necesario reconocer que las concepciones que emergen del discurso docente se sostienen también en un orden imaginario y enigmático inabordable en un contexto investigativo.

\section{Pensar y actuar en relación a la sexualidad. Concepciones docentes}

Siguiendo las conceptualizaciones que proponen Giordan y De Vecchi, entiendo por concepciones personales a la estructura conceptual en la que se insertan y organizan los conocimientos de los que se apropia un sujeto. Las mismas constituyen "una verdadera estrategia cognitiva que la persona pone en marcha para seleccionar las informaciones pertinentes, para estructurar y organizar lo real" (1987, p. 105). Las concepciones personales del docente en relación a la sexualidad se constituyen en una "estructura de recepción" que permite interpretar la realidad de una manera determinada. Dicha estructura 
Revista de la Escuela de Ciencias de la Educación, año 11, número 10, enero a diciembre de 2015. Páginas 201-214. ISSN 1851-6297. ISSN EN LINEA 2362-3349. LOS DISCURSOS EN TORNO A LA SEXUALIDAD. SU INTERNALIZACIÓN Y

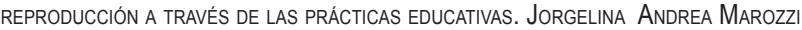

no se centra solamente en saberes conceptuales sino que está impregnada de prejuicios, estereotipos y componentes ideológicos. Esto es así ya que el sujeto está inserto al interior de tramas de relaciones sociales, se ubica desde pertenencias y prácticas sociales particulares que marcan su conocimiento de lo social. Esta alta cercanía sujeto-objeto impregna al conocimiento de lo social (aún más que a otros) de prejuicios, estereotipos y componentes ideológicos. Por lo tanto las creencias, sistemas de valores, prejuicios y estereotipos son parte constitutiva del "marco de referencia" del sujeto, al cual apela para construir sus concepciones sobre asuntos y problemas particulares (Bentolila y Rinaldi, 1998)

El docente, va configurando un propio posicionamiento, internalizando un determinado modo de ser/actuar/pensar/decir/sentir en torno a la sexualidad. Se trataría de un sujeto epistémico que va construyendo sus concepciones en un inter-juego entre asimilación-acomodación de las nuevas informaciones. Las concepciones entonces son producto de sucesivas reestructuraciones a lo largo de la historia del sujeto. Estas construcciones de conocimiento, integradas por informaciones, creencias, opiniones y actitudes, sirven como marco de percepción en la interpretación de la realidad, y muchas veces, guían las prácticas que realizan los distintos actores sociales.

Podemos pensar que la subjetividad de los docentes se fue constituyendo, como señalaba antes, en una sociedad en la que la sexualidad ha estado marcada históricamente por prohibiciones, silencios y ocultamientos, una sociedad donde existe una permisividad diferenciada entre los sexos, ocupando lo femenino un lugar de subordinación, una sociedad que niega el placer en sí mismo y concibe a la reproducción como fin del acto sexual (y en consecuencia silencia otras formas de manifestación de lo sexual), donde la experiencia sexual se halla teñida por connotaciones morales que definen las expresiones sexuales en torno al calificativo "bueno" o "malo".

Si éste es el mundo objetivo que se ha incorporado, es de esperar que éste sea el mundo que se seguirá reproduciendo. Como el habitus se manifiesta espontáneamente, sin conciencia ni voluntad, el rol docente puede convertirse en un rol reproductor de estereotipos sexuales. Los encargados de habilitar espacios en torno a la educación sexual, pondrán en juego sus propias concepciones acerca de la sexualidad, ya que "la propia subjetividad del adulto se convierte en el más valioso instrumento para la conformación de la subjetividad del niño" (Belgich, 2005 p.140).

Diversos estudios empíricos muestran esta relación. El Ministerio de Salud de la Nación, a través del Programa Nacional de Salud Sexual y Procreación responsable, realizó un proceso de investigación acerca de los enfoques predominantes en educación sexual, los cuales están íntimamente articulados con las concepciones sostenidas acerca de la sexualidad. La indagación mostró que existen enfoques que ponen el acento en una moral religiosa y, por ende, 
Revista de la Escuela de Ciencias de la Educación, año 11, número 10, enero a diciembre de 2015. Páginas 201-214. ISSN 1851-6297. ISSN EN LINEA 2362-3349. LOS DISCURSOS EN TORNO A LA SEXUALIDAD. SU INTERNALIZACIÓN Y REPRODUCCIÓN A TRAVÉS DE LAS PRÁCtICAS EdUCATIVAS. JoRgelina ANDREA MarozZI

sostienen una educación sexual orientada a favorecer la abstinencia o la postergación de las prácticas sexuales hasta edades más avanzadas, y otras perspectivas que entienden a la educación sexual como una instancia de prevención contra los riesgos que pueden traer las prácticas sexuales no responsables. Más allá de las diferencias que pueden encontrarse entre las distintas concepciones, son miradas que giran en torno a la "reproducción biológica" y al "peligro" de embarazos no planificados y de infecciones de trasmisión sexual. En las mismas "predomina una noción de sexualidad asociada a "naturaleza" humana y, por ende, es visualizada como una característica ahistórica que atraviesa todos los tiempos y las sociedades" (Canciano, 2007, p.14). En este marco, las diferencias sexuales se explican como desviaciones a la "regla" o a condiciones de "normalidad".

En concordancia con esto, Graciela Morgade (2005), como señalaba antes, identifica dos modelos dominantes en los programas de educación en la sexualidad: el modelo biologista, que se centra en lo anátomo-fisiológico, y el modelo moralizante, guiado por principios normativos que prescriben "lo que debe ser y no ser" en relación a la expresión de la sexualidad. Diversos estudios coinciden que la dimensión del deseo, del placer, permanece silenciado en la mayoría de los establecimientos (Fine, 1999; Epstein y Johnson, 2000, Hernández y Reybet, 2007; Pauluzzi, 2005). Investigaciones que indagan acerca de las visiones o concepciones sobre sexualidad que tienen docentes y directivos, acuerdan que impera hegemónicamente el discurso de la educación sexual para la "prevención" a fin de evitar los efectos "no deseados" de algunas prácticas. Esto destaca el carácter negativo que impregna la sexualidad, reduciendo a una concepción biologista y medicalizada que despoja a la misma del componente cultural (Pauluzzi, 2005; Sanchez Sosa, 2005; Hernández y Reybet, 2007ª). Algunos/as docentes minoritarios/as sostienen una concepción más amplia que incorpora el enfoque de género (Morgade, 2011, Fioretti y otras, 2004 -citado por Morgade-). Los maestros y profesores valoran positivamente que se brinde educación sexual en las escuelas (Jimenez Ríos, 2004; Universidad Nacional de Tres de Ferrero, 2006) pero a la vez manifiestan cierto temor a hablar del tema, "no saber"/"no poder", limitaciones por temor a las reacciones que pueda despertar entre los padres y madres de alumnos y por lo tanto, la recurrencia a especialistas para trabajar esta temática (Morgade, 2011; Hernández y Reybet, 2007a; Greco, 2007).

Por otra parte, los docentes visualizan una sola manera de construir la orientación de la sexualidad, conocido como el "mandato de la heterosexualidad obligatoria" y "heteronormatividad" (Alonso et all, 2008; Elizalde, 2009) que expresan un obstáculo para pensar la heterosexualidad como una institución política.

Un conjunto de investigaciones que aborda la cuestión de las representaciones atravesadas por cuestiones de género, ponen en evidencia los diferentes 
Revista de la Escuela de Ciencias de la Educación, año 11, número 10, enero a diciembre de 2015. Páginas 201-214. ISSN 1851-6297. ISSN EN LINEA 2362-3349. LOS DISCURSOS EN TORNO A LA SEXUALIDAD. SU INTERNALIZACIÓN Y REPRODUCCIÓN A tRAVÉS dE LAS PRÁCtiCAS EduCATIVAS. JoRgelina AndREA MarozZI

parámetros que utilizan los docentes para evaluar conductas en varones y mujeres, reproduciendo en su accionar estereotipos de género (Varela y Ferro, 2000; Pinkasz y Tiramonti, 2006; Tomasini, 2009). Así, existe un "curriculo oculto de género" (Dorr y Sierra, 1998) donde expectativas y valoraciones diferenciales para con sus alumnos y alumnas de parte de los docentes, se traducen en sus prácticas cotidianas de enseñanza (Palermo, 1996; Scharagrodsky, 1999; Hernández y Reybet, 2007b).

Hasta aquí podemos ver cómo las formas de entender a la sexualidad y a la educación sexual son producto de condiciones históricas, de estructuras objetivas que se interiorizan conformando estructuras subjetivas que se manifiestan en modos habituales de intervenir y enseñar en torno a la educación sexual. Pero también es importante tener en cuenta que los habitus aseguran la reproducción de las estructuras objetivas, siempre y cuando las condiciones en las que el habitus funciona sean semejantes a aquellas condiciones en las que el mismo se constituyó. Esto nos lleva a pensar que las prácticas están objetivamente inadaptadas a las condiciones presentes, ya que asistimos a una época de cambios vertiginosos, donde se habla más libremente de sexualidad en los espacios públicos, se sobrevalora la búsqueda del placer, la iniciación sexual de las mujeres solteras no resulta estigmatizada, las cada vez más precisas técnicas de fertilización asistida ponen en cuestión el fin reproductivo del acto sexual y hay una mayor aceptación de divorcios y concubinatos. Nos encontramos con un Estado que promueve políticas de reconocimiento, y donde los colectivos LGTBI han comenzado a recibir mayor aceptación por crecientes sectores sociales, conquistando derechos que se plasman en nuestro país en leyes como la Ley de Matrimonio Igualitario y la Ley de Identidad de Género.

En este marco social, las Organizaciones Internacionales adhieren a un concepto amplio de sexualidad, considerando a la misma como "una dimensión fundamental del hecho de ser humano. (...) Se expresa en forma de pensamientos, fantasías, deseos, creencias, actitudes, valores, actividades, prácticas, roles y relaciones. La sexualidad es el resultado de la interacción de factores biológicos, psicológicos, socioeconómicos, culturales, éticos y religiosos o espirituales" (OPS. OMS., 2002). Se sanciona una Ley de Educación Sexual Integral que entiende a la misma como un espacio de enseñanza y aprendizaje que promueve saberes y habilidades para la toma de decisiones conscientes y críticas en relación con el cuidado del propio cuerpo, las relaciones interpersonales, el ejercicio de la sexualidad y los derechos. Como "un tipo de formación que busca proporcionar herramientas de cuidado antes que modelar comportamientos" (Ministerio de Educación, 2007, p.14).

Esto interpela a los docentes a repensar las formas tradicionales en que se ha encarado la educación sexual en las escuelas. Los modelos biomédicos, que sólo trabajan la prevención de enfermedades, o los prescriptivos que se empeñan en definir conductas "correctas" o "inadecuadas", deben ser ahora 
Revista de la Escuela de Ciencias de la Educación, año 11, número 10, enero a diciembre de 2015. Páginas 201-214. ISSN 1851-6297. ISSN EN LINEA 2362-3349. LOS DISCURSOS EN TORNO A LA SEXUALIDAD. SU INTERNALIZACIÓN Y REPRODUCCIÓN A TRAVÉS DE LAS PRÁCtiCAS EduCATIVAS. JoRgelina ANDREA MarozZI

puestos en cuestión para pensar un nuevo abordaje centrado en los criterios que sustentan los Lineamientos Curriculares de Educación Sexual Integral: la promoción de la salud, la valoración de niños, niñas, y adolescentes como sujetos de derecho, el respeto por la diversidad, el rechazo a todas formas de discriminación, la igualdad de oportunidades para varones y mujeres, el desarrollo de competencias psicosociales, la afectividad, el conocimiento, valoración y cuidados del cuerpo humano, los valores, y la participación de los niños, niñas y adolescentes y de sus familias.

Podemos hipotetizar que el habitus de los docentes funciona en un mundo bastante distinto de aquel en que éste se fue construyendo, produciendo conflicto en los sujetos.

Frente a esto surgen algunos interrogantes ¿Podrá sostenerse en su discurso frente a un grupo de estudiantes un/a docente con un enfoque tradicional de la sexualidad? ¿Podrá ir modificando sus propios esquemas de apreciación, de valoración, teniendo en cuenta que los mismos tienen un carácter duradero capaz de "sobrevivir a las condiciones económicas y sociales de su propia producción"?

\section{Líneas de indagación}

Creo, a manera de hipótesis, que pueden suceder tres posibilidades. Una de ellas es que los/las profesores/as, de manera no consciente y como estrategia propia del habitus a fin de asegurar su constancia, solo se expongan a experiencias que refuercen el mismo y eviten aquellas que puedan ponerlo en cuestión. En tal caso, enseñando en instituciones educativas de estilo conservador, intentarán imponer un enfoque tradicional, reforzando prejuicios culturales, y estereotipos de género. Esta postura, creo puede producir en los/ las alumnos/as indiferencia, o un refuerzo de temores y sentimientos de culpa hacia la propia sexualidad que es vivida como amenazante o peligrosa. Por otra parte, si tenemos en cuenta que los cambios sociales van incidiendo en la constitución de la subjetividad, en la imagen de los cuerpos, planteando a los sujetos conflictos acerca de las propias creencias sobre su sexualidad, su manera de ser mujer y ser varón; podemos suponer que el posicionamiento docente en torno a la sexualidad y las prácticas de educación sexual que lleven a cabo, reproduzcan estos conflictos y confusiones, y el docente transmita las propias contradicciones que se le plantean. Se mezclaran entonces discursos que ligan lo sexual a la salud, combinando confusamente actitudes permisivas y moralizantes.

En el mejor de los casos, y teniendo en cuenta que el habitus es perdurable pero no inmutable, podrán los profesores, a través de un proceso de revisión de los propios temores acerca de la sexualidad a fin de desmitificar prejuicios sociales, y de historización a fin de vislumbrar los mecanismos de poder que 
Revista de la Escuela de Ciencias de la Educación, año 11, número 10, enero a diciembre de 2015. Páginas 201-214. ISSN 1851-6297. ISSN EN LINEA 2362-3349. LOS DISCURSOS EN TORNO A LA SEXUALIDAD. SU INTERNALIZACIÓN Y reproducCión a través de LAS pRÁcticas educativas. Jorgelina Andrea MarozZI

se ponen en juego para producir los discursos válidos sobre el sexo, revisar y modificar sus propias concepciones. También, a partir de la apropiación de conocimientos conceptuales, resignificar su propio posicionamiento, y transferir estos aprendizajes a sus prácticas docentes. Esta última posibilidad se sustenta entonces en la convicción de que los cambios que se están produciendo en la estructura objetiva de la sociedad respecto a lo sexual, inciden en las estructuras cognitivas de los sujetos, quienes, a partir de un proceso reflexivo pueden ir modificando los propios habitus, el propio posicionamiento. No basta con apropiarse de discursos pedagógicos renovadores, o elementos conceptuales, sino partir de una reflexión crítica y contextualizada de los propios supuestos, que permita reelaborar y ampliar los marcos interpretativos y de acción.

Estos supuestos -y otros-constituyen puntos de partida de un proceso de investigación actual que busca reconstruir las concepciones de los docentes en torno a la Sexualidad y a la Educación Sexual y su resignificación a partir de la participación en procesos de capacitación en Educación Sexual Integral. Se trata de conocer si un trabajo sistemático de revisión de tradiciones y costumbres incorporadas en los sujetos, a la luz de nuevas miradas, posibilita construir nuevos significados, nuevos marcos interpretativos que sirvan de sustento a prácticas de educación sexual integrales.

\section{Referencias bibliográficas}

- $\quad$ Ageno, R. (2000). "Análisis de la práctica educativa". En Laino, D. Aportes para una clínica del aprender. Rosario: Homo Sapiens Ediciones.

- $\quad$ Alonso, G., Herczeg, G., Zurbriggen, R. (2008). "Talleres de educación sexual, efectos del discurso heteronormativo". En Morgade, G. \& Alonso, G. Cuerpos y sexualidades en la escuela. De la "normalidad" a la disidencia. Buenos Aires: Paidós.

- Belgich, H. (2005). Los afectos y la sexualidad en la escuela (hacia una diversidad del sentir) Santa Fe: Homo Sapiens Ediciones.

- Bentolila, S., Rinaldi, M. (1998). Epistemología y Didáctica. Reflexiones en torno a algunos obstáculos en la enseñanza de las ciencias sociales para maestros. Alternativas, volumen (11) Publicación del Laboratorio de alternativas Educativas de la UNSL.

- $\quad$ Berger, P., Luckmann, T. (1989). La construcción social de la realidad. Buenos Aires: Amorrortu.

- $\quad$ Bourdieu, P. (1988). Cosas dichas. Buenos Aires: Gedisa.

- $\quad$ Bourdieu, P. (1991). El sentido práctico. Madrid: Taurus.

- Canciano, E. (2007). Indagaciones en torno a la problemática de la sexualidad en el terreno de la educación: relevamiento de antecedentes teóricos y programas de educación sexual. Buenos Aires: Ministerio de Salud de la Nación.

- Dorr, A., Sierra, G. (1998). El curriculum oculto de género. Educar: Revista de educación, nueva época (7). Guadalajara, México, Secretaría de Educación de Jalisco.

- Elizalde, S. (2009). Normalizar ante todo. Ideologías prácticas sobre la identidad sexual y de género de los/as jóvenes en la dinámica de las instituciones destinadas a la juventud. Revista Argentina de Estudios de Juventud, volumen (1)

- Epstein, D., Johnson, R. (2000). Sexualidades e Institución Escolar. Madrid: Morata. 
Revista de la Escuela de Ciencias de la Educación, año 11, número 10, enero a diciembre de 2015. Páginas 201-214. ISSN 1851-6297. ISSN EN LINEA 2362-3349. LOS DISCURSOS EN TORNO A LA SEXUALIDAD. SU INTERNALIZACIÓN Y

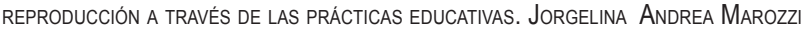

- $\quad$ Fine, M. (1999). "Sexualidad, educación y mujeres adolescentes. El discurso ausente del deseo". En Belausteguigoitia \& Mingo (Ed.) Géneros prófugos. Feminismo y Educación. México: Paidós.

- Foucault, M. (2002). Historia de la sexualidad. Argentina: Siglo XXI Editores.

- $\quad$ Foucault, M. (1996). La verdad y las formas jurídica. Barcelona: Gedisa.

- $\quad$ Giordan, A., De Vecchi, G. (1987). Los orígenes del saber. Paidós.

- Greco, B. (2007). Una escuela inclusiva de la sexualidad. Educación Sexual en la escuela. Perspectiva y reflexiones. Ministerio de Educación, Dirección General de Planeamiento. GCBA Buenos Aires.

- Hernández, A., Reybet, C. (2007). La/s sexualidades/es ¿tema de quiénes?. El Monitor de la educación, volumen (11)

- Hernández, A., Reybet, C. (2007). Géneros y sexualidades en el área de la Educación Física escolar. Aproximaciones a un estudio curricular comparado entre Canadá y la Argentina. Revista Argentina de Sociología, volumen (5) Buenos Aires.

- Jiménez Ríos, F. (2004). Formación sexual de profesionales de la educación. Un estudio experimental. Archivos Hispanoamericanos de Sexología, volumen (X).

- Ministerio de Educación de la Provincia de Córdoba (2007). Sexualidad y Escuela. Hacia una educación sexual integral. Documento base.

- Ministerio de Educación de la Provincia de Córdoba. Subsecretaría de Promoción de Igualdad y calidad. Dirección de Planeamiento e Información Educativa. Educación Sexual Integral. 2008.

- Ministerio de Educación. I/ Curso virtual de Educación Sexual Integral en la Escuela. Modulo 1 Introducción a los conceptos de sexualidad y de educación sexual, 2010. Argentina.

- Ministerio de Educación. Educación sexual Integral. Conceptualizaciones para su abordaje, 2010. Córdoba.

- Morgade, G. (2006). Educación en la sexualidad desde el enfoque de género. Una antigua deuda de la escuela. Novedades Educativas $\mathrm{N}^{\circ} 184$

- Morgade, G. (2011). Toda educación es sexual. Hacia una educación sexuada justa. Buenos Aires: La Crujía Ediciones

- Palermo, A. (1996). Concepciones de género de las maestras de escuelas primarias. Universidad Nacional de Luján.

- Pauluzzi, L. (2005). Educación Sexual y Prevención de la Violencia. Rosario: Hipólita Ediciones.

- $\quad$ Pinkasz, D., Tiramonti, G. (2006). Las oportunidades educativas de las mujeres en la modernización de los 90 en Argentina. Equidad de Género y Reformas educativas: Argentina. Chile. Colombia. Perú Santiago de Chile.

- Rubin, G. (1984). Reflexionando sobre el sexo: notas para una teoría radical de la sexualidad. Biblioteca virtual de Ciencias Sociales.

- $\quad$ Sanchez Sosa, I. (2005). Significado de la salud y la sexualidad en jóvenes. Un estudio de Caso en Escuelas Públicas de Cuernavaca. Instituto Nacional de Mujeres, México.

- $\quad$ Scharagrodsky, P. (1999). Cuerpo, género y poder en la escuela: La construcción de la masculinidad en las clases de Educación Física. Tesis de Maestría, FLACSO, Buenos Aires.

- Tenti Fanfani, E. (2005). La condición docente. Análisis comparado de la Argentina, Brasil, Perú y Uruguay. Buenos Aires: Siglo XXI Editores.

- Tomasini, M. (2009). El género como sistema de significación y la experiencia infantil. Un análisis de las marcas de género en los espacios y objetos de juego en ámbitos de educación inicial. Revista diálogos pedagógicos. Año VII N 14 Octubre/2009 
Revista de la Escuela de Ciencias de la Educación, año 11, número 10, enero a diciembre de 2015. PÁginas 201-214. ISSN 1851-6297. ISSN EN LINEA 2362-3349. LOS DISCURSOS EN TORNO A LA SEXUALIDAD. SU INTERNALIZACIÓN Y

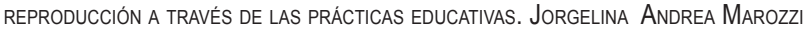

- Universidad nacional de Tres de Febrero y Ministerio de Educación del Gobierno de la Ciudad de Buenos Aires (2006). Consulta a docentes. Relevamiento de opinión sobre educación sexual en las escuelas. Buenos Aires.

- Varela B., Ferro L. (2000). Las Ciencias Sociales en el nivel inicial. Cap. III: Prácticas y representaciones habituales respecto del género en el nivel inicial. Ed. Colihue.

\section{Documentos}

- Ley Nacional N²6.150 Programa Nacional de Educación Sexual Integral. 2006 